\title{
Two levels of meaning elaboration in psychological research
}

Theory \& Psychology 202I, Vol. 3I(3) 446-450 (C) The Author(s) 2021

Article reuse guidelines: sagepub.com/journals-permissions DOI: I0.1 I77/095935432।I006780 journals.sagepub.com/home/tap

(3)AGE

\author{
David Dias Neto \\ ISPA - Instituto Universitário
}

\section{Mikael Leiman}

University of Eastern Finland

\begin{abstract}
Arocha (202I) discusses the "replication crisis," arguing for models that allow a greater complexity in the relationship between variables and processes. In this comment, we identify a more fundamental issue: the impossibility of eliminating interpretation issues with operational definitions and increased rigor in the measurements of variables and processes. Interpretation is at the core of (a) human action and (b) scientific endeavor. First, considering Vygotsky, we argue that all higher mental processes are sign mediated, influencing psychological research with humans. Second, that the understanding of research results also involves sign mediation and, therefore, it is nonneutral. We suggest two alternative approaches. There needs to be an increase in research that delivers a detailed description of psychological phenomena. Additionally, it is necessary to increase the elucidation of contextual-embeddedness research. Taking into account the two levels of meaning will underline psychology as a scientific discipline of complex phenomena.
\end{abstract}

\section{Keywords}

epistemology, replication crisis, semiotic mediation, variability, Vygotsky

In his article "Scientific Realism and the Issue of Variability in Behavior," Arocha (2021) makes several criticisms of traditional research in psychology. He starts from an ontological position of scientific realism, highlights problems in traditional research in psychology, and discusses two models that he considers to follow an alternative position. While we agree with Arocha on the criticisms of the traditional model, we argue that the alternatives proposed are insufficient. They do not consider that human activity,

\section{Corresponding author:}

David Dias Neto, ISPA - Instituto Universitário, Rua Jardim do Tabaco 42, Lisboa, I I00-08I, Portugal.

Email: dneto@ispa.pt 
including mental phenomena, is always directed towards objects (internal or external). Because such objects are referential sign formations, the issue of meaning and its interpretation cannot be bypassed. This inevitably affects the research methodology as well as the conceptual models of mental processes.

The issue of a crisis in psychology is not new. Almost a century ago, Vygotsky $(1927,1925 / 1997)$ wrote two rich papers addressing the causes and proposing a solution for the crisis in psychology. At the time, the crisis involved issues that have presently been set aside, such as the questions raised by participant self-report methods. Other issues remain relevant and are discussed within the current replication crisis: namely, the objectiveness of psychological measurement and the role of theory in framing observations (Vygotsky, 1927).

Vygotsky assumes two philosophical assumptions. Like Arocha (2021), he adopts an ontological realist stance. However, Vygotsky (1927) adds, as a second principle, the idea that reality is only understood through theory: "Whereas the first claimed that the highest scientific abstraction contains an element of reality, the second is the opposite theorem: even the most immediate, empirical, raw, singular natural scientific fact already contains a first abstraction" (p. 17). For Vygotsky, even naming an object is a theoretical action.

Vygotsky (1925/1997) proposes the consideration of psychology within two levels. The existing models or theories should be considered as applications of a more general psychology. This general psychology is thought of as a "unified scientific-objective system of methods for the investigation of and experiment with human behavior" (p. 12). This means that this general psychological science would be a broad theoretical and methodological umbrella for specific theories.

Arocha (2021) also proposes a theoretical solution for the crisis and provides two examples: perceptual control theory and observation-oriented modeling. Perceptual control theory is interesting because it deepens the relationship between input-output (e.g., Powers, 1978). However, it does not change the underlying understanding of the input nor the output (nor even the idea of control). Observation-oriented modeling is a novel and original approach to address individuals' variability (e.g., Grice, 2015). Nevertheless, it still aims to identify patterns in a manner that constitutes a nomothetic understanding of the relationship between variables. Both these theories deepen the relationship between variables, but both do not address how variables are created and how participants interact with the studied elements. Any theory that tries to account for psychic phenomena by abstracting its objects is inherently limited. These two theories, therefore, still constitute application theories within Vygotsky's framework. Vygotsky's proposal constitutes a more comprehensive alternative to the models proposed by Arocha (2021).

The subject matter of general psychology, proposed by Vygotsky (1927, 1925/1997), is sign-mediated activity. Given that meaning is intrinsically linked with language, it must be understood within Vygotsky's broader understanding of language and thought (Vygotsky, 2012). In language development, linguistic thought is intrinsically related to spoken language. Furthermore, for Vygotsky, as higher cognitive processes are internalizations of cultural practices, individual thought is intrinsically related to social processes. Sign and meaning bridge individual psychological processes with cultural and social dimensions. Considering these properties, sign-mediated activity is a useful unit for 
understanding and promoting a dialogue between theories as different as psychoanalysis and behaviorism.

\section{The role of interpretation in psychological research}

Variability in behavior results from human participants' interaction with a research apparatus (even a naturally occurring one) observed and interpreted by a researcher. Hence, two aspects or levels of interpretation are present in any scientific research with humans.

At the participant level, the consideration of meaning mediation provides a tool to understand participant interaction with the research apparatus. Some of what has been considered as bias has to do with this interaction. One example is the Hawthorne effect, which refers to changes in research participants' behavior due to the experimental observation. In the original studies (Gillespie, 1991; Landsberger, 1957), the researchers sought to observe increases in productivity by changing illumination. The researchers found an increase in productivity, irrespective of the change in illumination, and a subsequent decrease after the research observation had ceased. For positivistic research, the Hawthorne effect is considered a source of error in examining the effects of the variables. Vygotsky might have a different reading of these results. Signs mediate the response of workers to illumination changes or the observation by researchers. These signs are linked to meanings associated with engagement or resistance within the work context, which then influences productivity. More than constituting an error source, this consideration would increase our understanding of the phenomena.

The second aspect of interpretation involves researchers as individuals who interpret observations. Here, variability becomes a problem only when the researcher aims to generalize concrete and operationally defined behaviors. More than eliminating variability, the goal should be to provide scientific formulations of complex phenomena. One example is the fundamental attribution error, that is, the human tendency to attribute others' behavior to internal causes relative to situational factors. When this error was found not to be as universal as expected, this led to a greater understanding of the role of cultural practices in attribution (e.g., Mezulis et al., 2004; Miyamoto \& Kitayama, 2002). The loss in universality gave rise to a richer understanding of human attribution.

Participants actively interact with the research apparatus within a social and cultural medium. The consideration of this active contextual embeddedness is crucial in interpreting the results. Furthermore, the researchers also interpret the results within their social and cultural medium. Theories are such formulations. The idea of a general psychology that would provide a framework for specific psychologies or models would be a way to embrace this multiplicity of views. To achieve this, general psychology should frame psychology within a broad object and broad set of methods and admit the different levels of explanation that complex phenomena entail. A particular model would then be concrete applications to specific phenomena, alternative formulations to phenomena, or formulations that support applications of psychology. A general psychology would be a map to relate different theories or a medium to promote dialogue between formulations.

The so-called replication crisis seems to question psychology's claim as a science. Even today, the claim is supported by positivist procedures, that is, operational definitions, elimination of error, measurement procedures, and abolishing issues of meaning. 
These solutions actually generate the replication crisis. Arocha's (2021) proposal and the two examples mentioned in his article, despite being a step in the right direction, are still a step within this tradition. These two theories are applicable only to the implicit objects that fit the formalized process models proposed by the author. More than identifying or developing new models of behavior, it is important to frame and promote dialogue between existing ones. The consideration of the research participant as a human being (a semiotically mediated agent) and the researcher too as a human being increase the respect for complexity in psychology research. This respect implies the need for theoretical innovations, perhaps like Vygotsky's general psychology. Nevertheless, this respect for the complexity of human action is what will assert psychology as a scientific discipline.

\section{Author's note}

David Dias Neto is also affiliated with APPsyCI - Applied Psychology Research Center Capabilities \& Inclusion.

\section{Declaration of conflicting interests}

The authors declared no potential conflicts of interest with respect to the research, authorship, and/ or publication of this article.

\section{Funding}

The authors received no financial support for the research, authorship, and/or publication of this article.

\section{ORCID iD}

David Dias Neto (iD) https://orcid.org/0000-0002-3129-262X

\section{References}

Arocha, J. F. (2021). Scientific realism and the issue of variability in behavior. Theory \& Psychology, 31(3), 375-398. https://doi.org/10.1177/0959354320935972

Gillespie, R. (1991). Manufacturing knowledge: A history of the Hawthorne experiments. Cambridge University Press.

Grice, J. W. (2015). From means and variances to persons and patterns. Frontiers in Psychology, 6, Article 1007. https://doi.org/10.3389/fpsyg.2015.01007

Landsberger, H. (1957). Hawthorne revisited: A plea for an open city. Cornell University.

Mezulis, A. H., Abramson, L. Y., Hyde, J. S., \& Hankin, B. L. (2004). Is there a universal positivity bias in attributions? A meta-analytic review of individual, developmental, and cultural differences in the self-serving attributional bias. Psychological Bulletin, 130(5), 711-747. https://doi.org/10.1037/0033-2909.130.5.711

Miyamoto, Y., \& Kitayama, S. (2002). Cultural variation in correspondence bias: The critical role of attitude diagnosticity of socially constrained behavior. Journal of Personality and Social Psychology, 83(5), 1239-1248. https://doi.org/10.1037/0022-3514.83.5.1239

Powers, W. T. (1978). Quantitative analysis of purposive systems: Some spadework at the foundations of scientific psychology. Psychological Review, 85(5), 417-435. https://doi. org/10.1037/0033-295X.85.5.417

Vygotsky, L. (1927). The historical meaning of the crisis in psychology: A methodological investigation. In L. Vygotsky (Ed.), The collected works of Vygotsky (pp. 1-115). Plenum Press. 
Vygotsky, L. (1997). The methods of reflexological and psychological investigation. In R. W.

Rieber \& J. Wollock (Eds.), The collected works of L. S. Vygotsky: Vol. 3. Problems of the theory and history of psychology (pp. 1-20). Plenum Press. (Original work published 1925)

Vygotsky, L. (2012). Thought and language. MIT Press.

\section{Author biographies}

David Dias Neto, $\mathrm{PhD}$, is an assistant professor at ISPA-Instituto Universitário and a member of APPsyCI-Applied Psychology Research Center Capabilities \& Inclusion where he teaches and researches in clinical and health psychology, psychotherapy, and psychopathology. He was the former president of the clinical and health psychology division of the Portuguese Order of Psychologists. He has published several papers and books on psychotherapy process research, mental health, illness, and health perceptions.

Mikael Leiman, $\mathrm{PhD}$, is a professor emeritus in psychology, psychotherapy, and counselling at the University of Eastern Finland. He introduced cognitive analytic therapy in Finland and has trained several generations of psychotherapists over three decades. He is also a frequent visitor to the United Kingdom and other European countries, giving workshops on the semiotic understanding of mental actions and communication. Mikael is the developer of dialogical sequence analysis (DSA), a microanalytic research method aimed at exploring how internal mental activity is embodied in expression. It is mainly used in single-case psychotherapy and counseling studies. 